\title{
Contribución de las Prácticas Profesionales en la formación de los Estudiantes de la Facultad de Ingeniería Química de la Universidad Autónoma de Yucatán (México)
}

\author{
Miriam V. Chan-Pavon, Daniel A. Mena-Romero, Jesús F. Escalante-Euán, María D. Rodríguez-Martín \\ Universidad Autónoma de Yucatán, Facultad de Ingeniería Química, Periférico Norte Kilómetro 33.5 Tablaje \\ Catastral 13615 Chuburna de Hidalgo Inn C.P. 97203, Mérida, Yucatán, México \\ (e-mail: cpavon@correo.uady.mx; mromero@correo.uady.mx; jesus.escalante@correo.uady.mx; \\ dalmira.rodriguez@correo.uady.mx)
}

Recibido Abr. 28, 2017; Aceptado Jul. 3, 2017; Versión final Ago. 24, 2017, Publicado Feb. 2018

\begin{abstract}
Resumen
La investigación tuvo como objetivo identificar la satisfacción de los estudiantes de tres de los cinco programas de licenciatura de la Facultad de Ingeniería Química de la Universidad Autónoma de Yucatán (México) en la realización de las prácticas profesionales. El estudio permite también inferir si sus empleadores conocen las competencias de la licenciatura, con lo que se validaría a la práctica profesional como un escenario real de aprendizaje y de experiencia laboral. La investigación consistió en un estudio no experimental de diseño transversal para un grupo de estudiantes en un solo momento temporal en el marco de la asignatura de prácticas profesionales. En los resultados se destaca que la realización de las prácticas profesionales ha mejorado la vinculación entre la universidad y las empresas. Al tener escenarios reales de aprendizaje el $96 \%$ de los estudiantes manifestaron que aplicaron los conocimientos adquiridos en el salón de clases, un $84 \%$ manifestó haber adquirido nuevos conocimientos de valor curricular para su formación y las actividades que realizaron fueron de provecho para la empresa.
\end{abstract}

Palabras clave: prácticas profesionales; competencias; vinculación; mercado laboral; escenarios reales de aprendizaje

\section{Contribution of the Internship in the Professional formation in the Students of Chemical Engineering Faculty at the Autonomous University of Yucatan (México)}

\begin{abstract}
The aim of the research was to identify the satisfaction of students in three of the five undergraduate programs of the Faculty of Chemical Engineering of the Autonomous University of Yucatan (Mexico) in the experience obtained during their professional internships. The study also allows to inferring whether their employers know the competences of the degree, as to validate the internship as a real scenario of learning and acquisition of work experience. The research consisted of a non-experimental study of cross-sectional design for a group of students in a single temporary moment in the internship course. The results highlight that the implementation of internship has improved the university-industry collaboration allowing real learning scenarios. Of all the students involved in the study, $96 \%$ stated that they applied the knowledge acquired in the classroom, $84 \%$ stated that they had acquired new knowledge of curricular value during training and that the activities performed were of benefit to the company.
\end{abstract}

Keywords: internship; competences; collaboration; labor market; real learning scenarios 


\section{INTRODUCCIÓN}

Sin lugar a dudas, la formación práctica de un estudiante universitario es de gran relevancia tanto para el desempeño de la profesión como para su desarrollo personal y académico (Oyola y Padilla, 2012). En esta formación, destaca particularmente las prácticas en empresas, por ser un momento de "iniciación" en la vida profesional, así como por la duración temporal que posee en una determinada titulación. Las prácticas se convierten así en una situación de aprendizaje, una experiencia personal y profesional al mismo tiempo que es un componente curricular más, una parte sustantiva y obligada en gran parte de las titulaciones en la educación superior (Raposo y Zabalza, 2011). Polaino y Romillo (2017) hablan sobre la importancia de la vinculación de la Universidad con la sociedad y describen los tres componentes fundamentales: capacitación, consultorías-prestación de servicios y por último la práctica pre-profesional que es en donde se inserta al estudiante.

La práctica profesional integra al estudiante en un contexto de aprendizaje situado en escenarios reales de aprendizaje, relacionados con la práctica del rol profesional a desempeñar, posibilitando la adquisición de conocimientos, habilidades y competencias necesarias para el ejercicio profesional. No sólo se movilizan competencias adquiridas en la escuela, sino que se aprenden nuevos conocimientos y nuevas formas de abordar los problemas a partir de situaciones reales, con frecuencia en ambientes complejos e inciertos (Carey y Vargas, 2016). "Carey y Vargas (2016) en su trabajo La residencia profesional en Ingeniería Logística: Una aproximación al entorno laboral menciona que el panorama empresarial y de mercado junto con los cambios sociales, ofrece unas singularidades que, logicamente vienen a insidir en el perfil del profesional que debe egresar de nuestras aulas (Ruiz Ojeda, 2010)".

Las empresas en búsqueda de cumplir con esos cambios y ser cada día más competitivos, requieren de personal altamente calificado para poder satisfacer estas necesidades, por lo cual requieren la colaboración del sector universitario, para poder formar los perfiles que las empresas de hoy requieren. Según González y Ramírez (2011) las competencias laborales se basan en las cualidades de las personas para desempeñarse productivamente en una situación de trabajo, no sólo dependen de las situaciones de aprendizaje escolar formal, sino también del aprendizaje derivado de la experiencia en situaciones concretas de trabajo, son patrones que permiten comprobar si un trabajador es competente o no.

Para lograr formar profesionales con estas competencias, se deben considerar las estrategias educativas a utilizar, los contenidos, los objetivos del programa y los resultados del aprendizaje, las experiencias educativas, el entorno educativo, la evaluación, los estilos y ritmos de aprendizaje y la programación de tareas (Palés, 2006). La Universidad Autónoma de Yucatán (UADY), en respuesta a esta necesidad de los mercados globales, hace énfasis en la capacitación y formación profesional constante de los estudiantes, a través de sus programas de licenciatura, que se imparten en la Facultad de Ingeniería Química (FIQ), a través de iniciativas que coadyuven a incorporar a los estudiantes en escenarios reales de aprendizaje. Es ahí donde el profesionista formado en la FIQ puede contribuir al aumento de la competitividad de las empresas al insertarse en el área laboral una vez egresado.

En el contexto de la FIQ, los estudiantes requieren tener una visión clara de cuál es su mercado laboral y cómo su participación y desempeño en el programa de prácticas profesionales, junto con los conocimientos enseñados en la currícula de materias pudiera impactar en su desarrollo profesional a corto y largo plazo, es por eso que se requiere identificar la contribución de las prácticas profesionales en la formación de los estudiantes de las licenciaturas en Ingeniería Industrial Logística, Ingeniería Química Industrial y Química Industrial, impartidas durante el período de enero a diciembre de 2013. Hernández et al. (2012) señalan que recabando información acerca del progreso de los egresados, se puede obtener información valiosa para ganar terreno en aspectos como: fortalecer los programas de estudio y la práctica docente.

Mésquita et al. (2013) encontraron que desde el punto de vista de los profesionales es relevante, una enseñanza con base en escenarios reales. El estudio también hace énfasis en la importancia del enfoque de escenarios reales de aprendizaje para mejorar la calidad de una currícula de materias de ingeniería y el desarrollo de tanto competencias técnicas como transversales. Eskandari et al. (2007) identificaron que es muy importante revisar la currícula de materias, debido a los cambios que se dan en los roles y responsabilidades que debe tener un ingeniero y que para mejorar los resultados de la educación se deben establecer procesos iterativos y continuos para ser evaluada. También, Goñi y Meseguer (2010) señalan que se deben renovar las metodologías docentes partiendo de las competencias concretas de cada grado universitario. Las herramientas de que dispone para ello son variadas, pero todas ellas exigen un cambio de mentalidad y un esfuerzo añadido de trabajo al profesor, sin perder de vista, claro está, el objetivo que se pretende en la planificación por competencias, que es precisamente centrar el objetivo en el proceso de aprendizaje en el estudiante, en un marco que se extiende ahora a lo largo de la vida. 
Según Quispe et al. (2014) el desconocimiento de los estudiantes sobre los requisitos y características de las prácticas profesionales, pudieran ocasionar un posicionamiento laboral no adecuado para las características profesionales de los mismos durante su participación en el programa de prácticas profesionales, generando así el desaprovechamiento de la primera experiencia laboral para muchos de los mismos. De igual forma la falta de apoyo de los empleadores pudiera dificultar la experiencia de los estudiantes durante el periodo de prácticas profesionales. Martínez, (2003) señala que la inadecuación entre la formación del universitario y las tareas que desempeñan durante prácticas ha ocurrido en varias ocasiones. El desconocimiento de algunos empresarios sobre las competencias profesionales para las que preparan diferentes titulaciones ha hecho que muchos universitarios desempeñen cometidos inadecuados para su formación y, en otras ocasiones, alejados de sus posibilidades y de su responsabilidad. A su vez Villordo, (2001) encontró que las principales causas de fallas de los proyectos asignados a los alumnos pueden resultar difíciles de realizar debido a la gestión de tiempos, proponen metas demasiado ambiciosas y no son planeados adecuadamente.

Se quiere identificar el grado de satisfacción de los estudiantes de las licenciaturas de la FIQ al realizar sus prácticas profesionales e inferir si sus empleadores conocen el alcance que tiene la licenciatura que se encuentran cursando, así como la importancia de su realización en los estudiantes y la prioridad de los factores involucrados para el éxito en el desarrollo de la misma. Se pretende posteriormente realizar un análisis para identificar áreas de oportunidad para mejorar las prácticas profesionales. El presente estudio consideró a estudiantes de las licenciaturas en Ingeniería Química Industrial, Ingeniería Industrial Logística y Química industrial de la UADY que realizaron sus prácticas profesionales durante el periodo comprendido de enero a diciembre de 2013.

\section{METODOLOGÍA}

La presente Investigación fue de tipo descriptiva y exploratoria ya que tuvo como propósito identificar la contribución de las Prácticas Profesionales en la formación de los estudiantes de la FIQ.

\section{Diseño de la Investigación}

El presente estudio es una investigación no experimental de diseño transversal, ya que mide las variables de estudio en un solo momento temporal, la obtención de los datos se realizará una sola vez en cada unidad de análisis, y se utilizará un solo instrumento de recolección de información, con aplicación única a cada sujeto de investigación.

\section{Población}

La población objeto de estudio fueron los 74 estudiantes de la FIQ de las licenciaturas: Ingeniería Química Industrial, Ingeniería Industrial Logística y Química Industrial que se encontraban cursando el Módulo o Taller de Experiencia en el Trabajo.

\section{Fuentes para la obtención de la Información}

La información fue obtenida a través de lo que manifestaron los estudiantes en el instrumento (encuesta) que se aplicó, lo que permitió obtener la información que se quería recabar.

\section{Instrumento de Investigación}

Se utilizó una encuesta, previamente validada por un grupo de expertos locales y piloteada con 8 alumnos, que constó de 6 secciones la cual fue elaborada mediante la herramienta de formularios de GoogleDrive@ de manera estructurada y se administró mediante uso de correo electrónico a todos los estudiantes de la población de nuestra investigación. Toda la información recabada fue almacenada en la base de datos de esta herramienta para su análisis.

La encuesta permaneció abierta durante cuatro semanas y fue aplicada en línea para permitir que los alumnos pudieran llenarla con respuestas razonadas y cuando ellos dispusieren de tiempo.

\section{RESULTADOS Y DISCUSION}

Los resultados se presentan considerando los distintos aspectos presentados y analizados en este trabajo: datos generales, la empresa, satisfacción, conocimientos aplicados, contribución de las prácticas profesionales, y opiniones y sugerencias 


\section{Datos Generales}

El objetivo de esta sección fue obtener información general de nuestra muestra de estudio como carrera, sector en el que están realizando sus prácticas, tamaño de la empresa y motivación por las prácticas profesionales. La población de alumnos en las licenciaturas está distribuida de la siguiente manera (fig.1): 47 estudian Ingeniería Industrial Logística (IIL), 23 Ingeniería Química Industrial (IQI) y 4 de Química Industrial (QI).

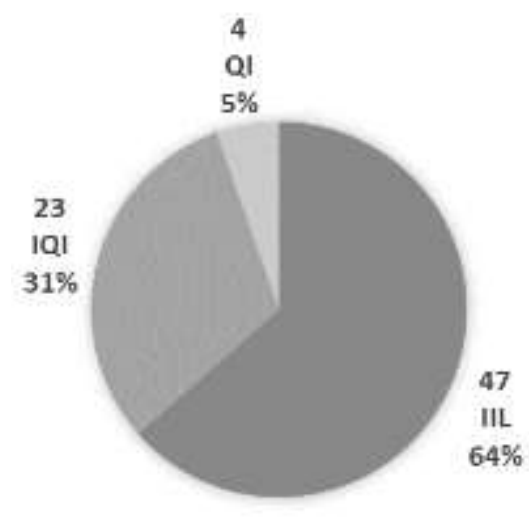

Fig. 1: Proporción de estudiantes realizando prácticas profesionales por licenciatura.

En la fig. 2 se aprecia el sector donde se encuentran realizando sus prácticas profesionales, observando que la mayor parte de los estudiantes se encuentra en el ramo industrial teniendo a 39 personas es este rubro equivalente al $53 \%$, y el $47 \%$ se encuentra en el ramo comercial, servicios y otros.

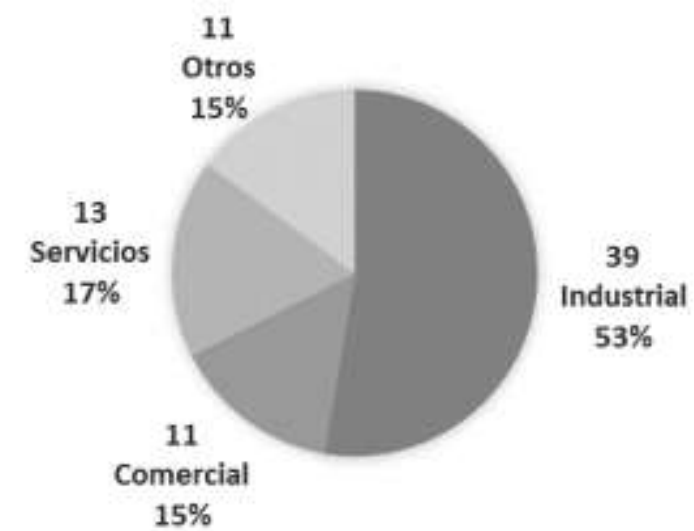

Fig. 2: Sector en el que se realizan las prácticas profesionales.

En la fig. 3 se aprecia cómo están distribuidos los alumnos en las empresas de acuerdo al número de empleados que estas tienen, se observa que existe una proporción muy similar, $54 \%$ se encuentran en empresas que tienen de 101 a 250 empleados y el $46 \%$ en empresas de 1 a 100 empleados.

22

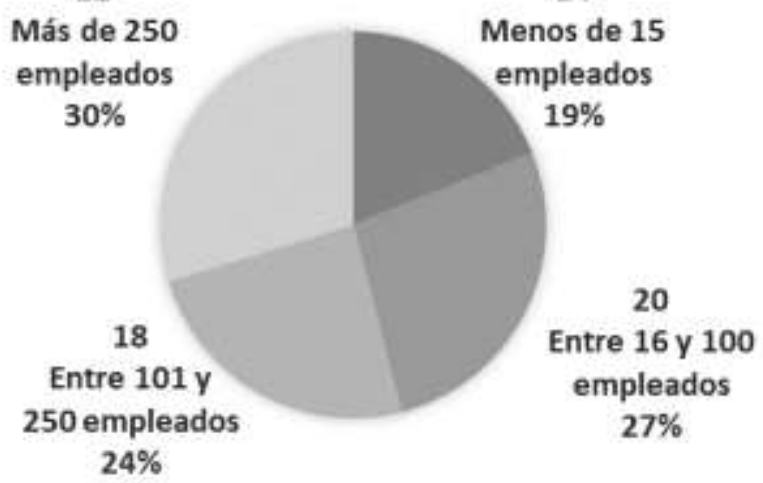

Fig. 3: Tamaño de la empresa según el número de empleados donde se encuentran los alumnos. 
En la tabla 1 se muestran los principales motivos por los que los alumnos realizan las prácticas profesionales observándose que la mayoría las realizan por el aprendizaje y la adquisición de experiencia profesional, con lo que demuestran comprender el objetivo de las mismas ya que no priorizaron el hecho de que es un requisito obligatorio para la titulación.

Tabla 1: Motivo de realización de las prácticas profesionales

\begin{tabular}{|l|c|c|}
\hline Motivo de Realización de Prácticas Profesionales & Frec. & $\%$ \\
\hline Aprender conocimientos del mundo laboral & 32 & $43.2 \%$ \\
\hline Adquirir valor curricular & 27 & $36.5 \%$ \\
\hline Adquirir experiencia profesional & 12 & $16.1 \%$ \\
\hline Porque es un requisito académico para la titulación & 1 & $1.4 \%$ \\
\hline Obtener una forma de ingresos económicos & 1 & $1.4 \%$ \\
\hline Otro & 1 & $1.4 \%$ \\
\hline
\end{tabular}

\section{La Empresa}

En esta sección se muestran los principales motivos de selección de la empresa en donde se realizan las prácticas profesionales, donde cerca de la mitad (42\%) escogió donde realizar sus prácticas de entre varias opciones laborales y otro porcentaje considerable $(28 \%)$ escogió el lugar gracias a recomendaciones de amigos, familiares o conocidos. Un porcentaje bajo $(7 \%)$ no pudo escoger el lugar, debido a que solamente fue seleccionado por una empresa. Un factor que casi no tuvo importancia (4\%) para los alumnos fue el atractivo del apoyo económico que ofrecía la empresa como se muestra en la tabla 2.

Tabla 2: Frecuencia y porcentaje del motivo de selección de la empresa

\begin{tabular}{|l|c|c|}
\hline Motivo de Selección de Empresa & Frec. & $\%$ \\
\hline Porque es la que yo seleccione, entre varias & 31 & $42 \%$ \\
\hline Porque fue donde me recomendó una persona & 21 & $28 \%$ \\
\hline Porque es la que me selecciono & 9 & $12 \%$ \\
\hline Porque es la única que me selecciono, pero no era de mi preferencia & 5 & $7 \%$ \\
\hline Otro & 5 & $7 \%$ \\
\hline Porque el apoyo económico me pareció atractivo & 3 & $4 \%$ \\
\hline
\end{tabular}

Con respecto a las actividades asignadas en la empresa (fig. 4), el $41 \%$ de los alumnos opinó que éstas tienen de 81 a $100 \%$ de relación con la licenciatura que estudian y un $28 \%$ manifestó que tienen una relación de un 61 a $80 \%$, este dato es de suma importancia ya que el propósito de las prácticas profesionales es que los alumnos apliquen los conocimientos adquiridos durante su formación académica, lo cual demuestra que las empresas tienen conocimiento de las áreas de competencia de los practicantes.

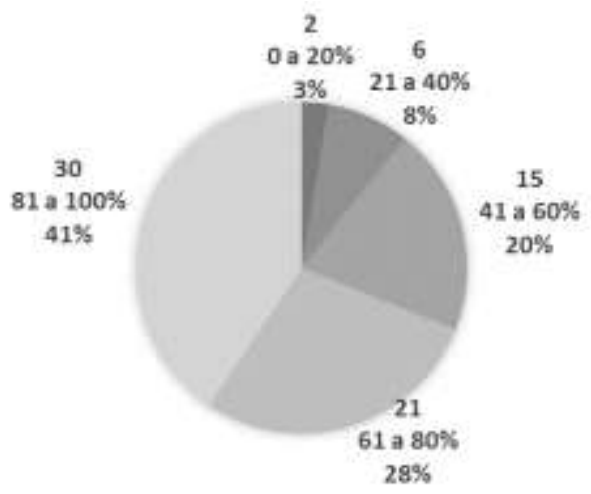

Fig. 4: Aplicación de los conocimientos adquiridos en la licenciatura en sus prácticas profesionales. 


\section{Satisfacción}

La finalidad de esta sección fue conocer el grado de satisfacción de los estudiantes respecto a las actividades realizadas y al ambiente laboral de la empresa. En la fig. 5 , se puede observar que en cuanto al ambiente laboral el $64.9 \%$ opinó que siempre se sintió a gusto en la empresa, el $50 \%$ manifestó que siempre sus recomendaciones se tomaron cuenta y el $90.5 \%$ opinó que siempre o la mayoría de las veces se sintió a gusto con las actividades desempeñadas. En general, los alumnos se sintieron a gusto en la empresa donde realizaban sus prácticas profesionales ya que percibieron que el ambiente era positivo y los jefes escuchaban y motivaban a los practicantes haciéndolos sentir parte de la misma. Es de resaltar que el $14.9 \%$ de los practicantes opinaban que algunas veces no coincidía la idea que tenían sobre las prácticas profesionales y lo que estaban haciendo en ellas, con lo que es recomendable investigar si esto es debido a falta de información por parte de la escuela o que están realizando en la empresa actividades no contempladas en su formación.

¿Te sientes a gusto con las actividades que realizas en tus PP?

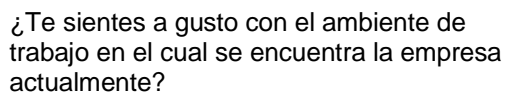

¿Te sientes a gusto con el ambiente de trabajo en el cual se encuentra la empresa actualmente?

¿Han tomado en cuenta tus opiniones y recomendaciones?

¿Siempre estas ocupado realizando tus labores como practicante?

¿Se te es difícil la comunicación con tu jefe?

¿Tu jefe inmediato te motiva o da buenas opiniones acerca de tu labor como practicante?

¿Los empleados te tratan como una persona importante dentro de la empresa?

¿La idea que tenías sobre las PP tiene relación con lo que estás haciendo en ellas?

\section{- Siempre = La mayoria de las veces =Algunas veces = Nunca}

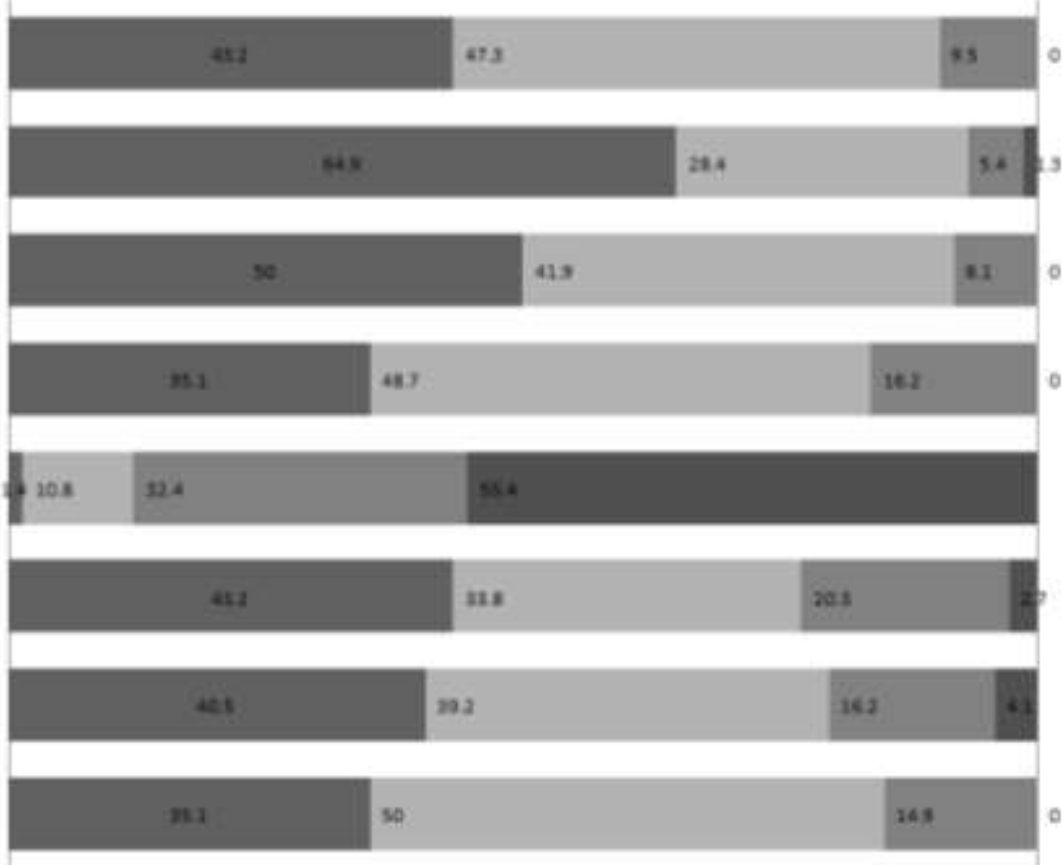

Fig. 5: Grado de satisfacción de los alumnos.

\section{Conocimientos Aplicados}

Esta sección es de gran importancia porque permitió obtener información sobre los conocimientos que se aplicaron en la empresa, si estos fueron los adquiridos en su formación académica, si son únicamente conocimientos de alguna materia específica, si lo que se ha aprendido hasta el momento de aplicar la encuesta fue clave para el desempeño dentro de la empresa y si se adquirió algún conocimiento nuevo en la empresa donde se realizó la práctica profesional. En las figuras 6 y 7 se puede observar que de los 74 alumnos evaluados el $96 \%$ de ellos afirmó que aplica los conocimientos adquiridos en su formación académica en las distintas actividades desempeñadas en sus prácticas profesionales y el $84 \%$ ha adquirido nuevos conocimientos para su formación profesional en la empresa, con lo que se cumple el doble propósito de las prácticas profesionales.

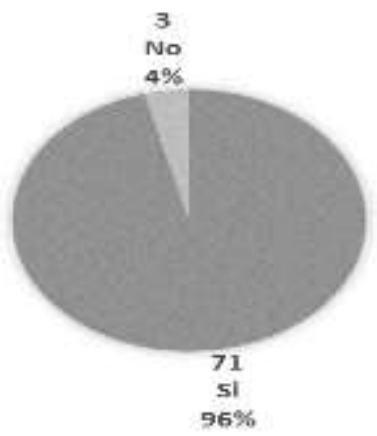

Fig. 6: Aplicación de conocimientos adquiridos en su formación académica. 


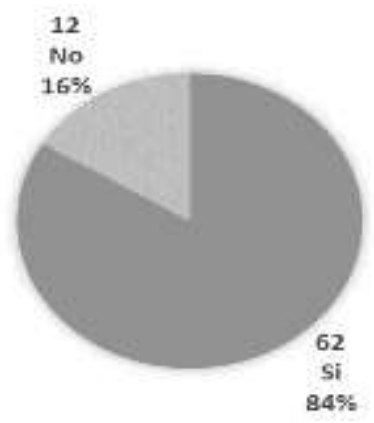

Fig. 7: Adquisición de conocimientos nuevos durante las prácticas profesionales.

\section{Contribución de las prácticas profesionales}

Esta sección se dividió en dos partes: Actividades y Factores de importancia para la realización de las prácticas. En la fig. 8 se enlistan las actividades, tomando en cuenta procesos operativos y actividades que tienen que ver con el control de calidad, supervisar maquinas o personal, generar pedidos, etc.). Se utilizó una escala Likert (Likert, 1932), que comprende desde "siempre" hasta "nunca", donde se puede observar que las actividades que tuvieron la calificación de "siempre" con mayor frecuencia son procesos operativos básicos como: registrar datos con una frecuencia de $48 \%$, llenar formatos con $39 \%$ y elaboración de reportes e informes con la máxima frecuencia de $49 \%$; también se puede observar que las actividades de archivar documentos y actividades de calidad son de las que con mayor frecuencia desempeñan los estudiantes en las empresas, así como que ellos no están involucrados en actividades de toma de decisiones estratégicas para la organización ni en mandos altos como tampoco en actividades al nivel de obrero.

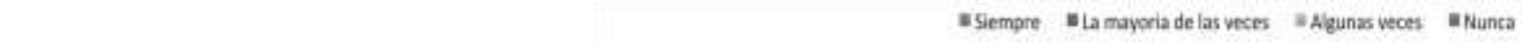

Participar en el análisis interno de la organización para identificar áreas de

Participar en el análisis externo de la organización

Realizar actividades relacionadas con el control de calidad

Asesorar a nivel directivo para el desarrollo y/o implementación de nuevos proyectos.

Identificar y tomar acciones sobre problemas operativos

Identificar necesidades de capacitación

Definir indicadores para el área de trabajo

Elaborar reportes e informes

Supervisar el flujo de materiales, equipo y/o transporte

Registrar datos

Operar máquinas y equipos

Implementar programas de mantenimiento preventivo y correctivo del área de trabajo

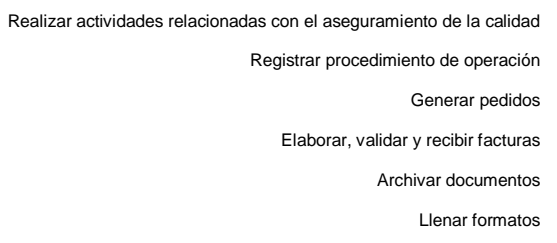

Supervisar al personal para la realización de su trabajo

Participar en labores que son realizados fuera de la empresa

Medir tiempos y movimientos de los empleados

Desarrollar nuevos productos

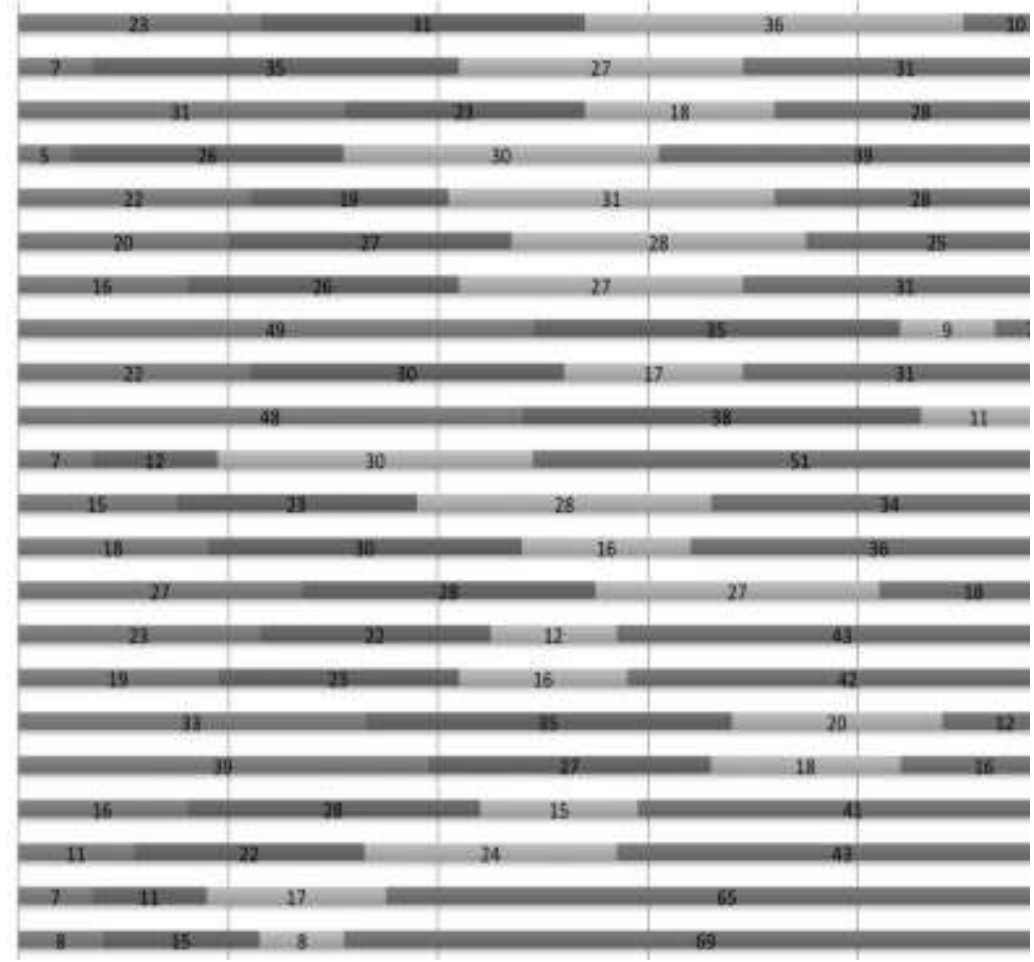

Fig. 8: Actividades que desempeñan los alumnos en las prácticas profesionales.

La segunda parte nos proporciona información sobre: la importancia de las prácticas profesionales, los valores que como estudiantes consideran más importantes para desempeñar su trabajo en la empresa, y los elementos necesarios para el desempeño de las actividades a realizar. En la tabla 3, se muestra los resultados numéricos, siendo el factor más determinante para lograr el éxito la actitud del estudiante para enfrentarse a los retos que puedan surgir con un $89 \%$, los valores ocupan el segundo lugar con $58 \%$ y los conocimientos con que cuentan ocupan las últimas posiciones. Los conocimientos básicos se refieren a física, matemáticas o química, los técnicos a aquellos que están relacionados con su carrera y los específicos a áreas de especialización dentro de su carrera. Así mismo mencionaron como valores más importantes la responsabilidad, la honestidad y el respeto. 
Tabla 3: Factores de importancia para el éxito del desarrollo de las prácticas profesionales en la empresa.

\begin{tabular}{|l|c|c|}
\hline Factores & Frecuencia & $\%$ \\
\hline Actitud & 66 & $89 \%$ \\
\hline Valores & 43 & $58 \%$ \\
\hline Conocimientos Básicos & 40 & $54 \%$ \\
\hline Conocimientos Técnicos & 31 & $42 \%$ \\
\hline Conocimientos Específicos & 30 & $40 \%$ \\
\hline
\end{tabular}

Por último, en la fig. 9, se muestra la apreciación que los estudiantes tienen sobre la importancia de la realización de las prácticas profesionales en su formación profesional; de manera que 58 de ellos que representan el $78 \%$ indicaron que esta actividad (asignatura obligatoria con el mayor de los créditos en la malla curricular) es muy importante, 16 alumnos representando el $22 \%$ consideran que es importante y ninguno las consideró poco o nada importante.

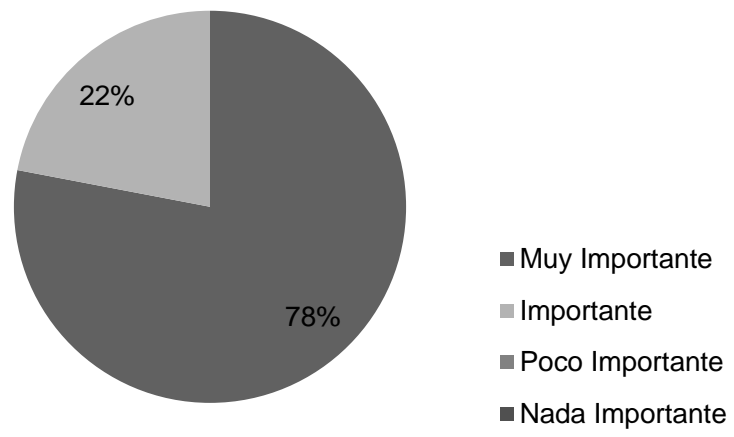

Fig. 9: Importancia de las prácticas profesionales en la formación profesional.

\section{Opiniones y Sugerencias}

La finalidad de esta sección fue la búsqueda en la mejora del proceso de prácticas de los alumnos, así como mejorar el proceso de formación de los alumnos. Por lo que se realizaron unas preguntas de retroalimentación, en donde los alumnos pudieron proponer cambios para mejorar las prácticas profesionales, según las experiencias que vivieron. En cuanto a las acciones de mejora que se obtuvieron, la mayoría de los estudiantes opinó que se dé una mayor difusión a las vacantes de prácticas profesionales con las que cuentan las empresas con un $82 \%$, seguido de pláticas por parte de los alumnos que ya concluyeron sus prácticas, donde puedan contar sus experiencias y dar consejos para poder evitar errores o mal entendidos que puedan suceder a los nuevos practicantes; las demás opiniones se aprecian en la tabla 4.

Tabla 4: Sugerencias para mejorar las prácticas profesionales en la FIQ según los alumnos

\begin{tabular}{|c|c|c|}
\hline Acciones de mejora & Frecuencia & $\%$ \\
\hline Dar mayor difusión a vacantes de empresas & 61 & $82 \%$ \\
\hline Pláticas de alumnos que ya hayan realizado prácticas & 52 & $70 \%$ \\
\hline $\begin{array}{l}\text { Cursos de formación profesional (elaboración de currículo, } \\
\text { talleres de liderazgo, etc.) }\end{array}$ & 49 & $66 \%$ \\
\hline Reducir el número de créditos para poder cursar la asignatura & 40 & $54 \%$ \\
\hline Que empresas formalicen un programa de practicantes & 32 & $43 \%$ \\
\hline Aclarar requisitos documentales para aprobar la asignatura & 7 & $9 \%$ \\
\hline
\end{tabular}

\section{CONCLUSIONES}

Los alumnos están conscientes de la importancia de las prácticas profesionales en su formación profesional y las realizan principalmente motivados por aprender conocimientos del mundo laboral, el valor curricular y para adquirir experiencia profesional. Ellos eligen el lugar donde las realizan de acuerdo a sus criterios acerca de la empresa y no por el apoyo monetario o por la falta de opciones en el mercado laboral. 
Las actividades que realizan en la empresa tienen relación con los conocimientos adquiridos durante su formación académica con lo cual se cumple el objetivo de las prácticas profesionales de que los alumnos adquieran experiencia de lo aprendido en el aula. La malla curricular de estudios de las licenciaturas, preparan a los alumnos con los suficientes conocimientos para desenvolverse de manera satisfactoria dentro de las empresas, siendo las asignaturas de gestión y planeación las más utilizadas y demandadas por los alumnos, a pesar de que la mayoría de los alumnos realizan actividades de tipo operativo básico con bastante frecuencia.

Se pudo apreciar en los resultados obtenidos que los alumnos se sienten satisfechos con respecto a las actividades que realizan, con el ambiente laboral, y con el trato de sus jefes inmediatos.

De las opiniones que los alumnos emitieron para la mejora del proceso de realización de las prácticas profesionales se resalta que debe de darse más difusión a las vacantes de las empresas y que consideran que se debe prestar mayor énfasis al proceso previo, donde los compañeros que ya han concluido con sus prácticas profesionales podrían aportar su experiencia y dialogar con sus compañeros de semestres inferiores, para orientarlos respecto al proceso, recomendar y orientar a los compañeros sobre las empresas donde pueden realizar sus prácticas, con base en la experiencia y conocimiento de las mismas.

\section{REFERENCIAS}

Carey, C. y Vargas, M., La residencia profesional en Ingeniería Logística: Una aproximación al entorno laboral, https://goo.gl/HXqKR1, ISSN: 2395-9878, Revista Electrónica ANFEI Digital, 2(4), 1-10 (2016)

Eskandari, H., Sala-Diakanda, S. y otros cuatro autores, Enhancing the undergraduate industrial engineering curriculum: Defining desired characteristics and emerging topics, doi: 10.1108/00400910710729875, Education + Training, 49(1), 45-55 (2007)

González, M. y Ramírez, I., La formación de competencias profesionales: un reto en los proyectos curriculares universitarios, https://goo.gl/Sa62mH, ISSN: 1870-1477, Odiseo Revista electrónica de pedagogía, 8(16), 1-12 (2011)

Goñi, M. y Meseguer, S., Diseño Curricular Centrado en las Competencias que debe Adquirir el Estudiante del Grado en Derecho, doi: 10.4067/S0718-50062010000200006, Form. Univ. [online], 3(2), 37-46 (2010)

Hernández, C. A., Tavera, M. E. y Jiménez M., Seguimiento de Egresados en Tres Programas de Maestría en una Escuela del Instituto Politécnico Nacional, doi: 10.4067/S0718-50062012000200006, Form. Univ. [online], 5(2), 41-52 (2012)

Likert, R., A Technique for the Measurement of Attitudes, in Archives of Psychology No. 140 by R.S. WOODWORTH Editor, 22, 5-55, New York, USA (1932)

Martínez, R., La inserción laboral de los universitarios a través de las prácticas en empresas. https://goo.gl/73Ep8Z, ISSN: 0210-5233, Reis. Revista Española de Investigaciones Sociológicas [en línea], (101), 229-254 (2003)

Mesquita, D., Lima, R.M. y Flores, A., Developing professional competencies through projects in interaction with companies: A study in Industrial Engineering and Management Master Degree, $5^{\text {th }}$ International Symposium on Project Approaches in Engineering Education, 103.1-103.7, Eindhoven, Holland, 8-9 de Julio (2013)

Oyola, M.A. y Padilla, L.M., El reto frente a la globalización: la competitividad desde un enfoque sistémico, doi: 10.21500/01235834.639, Gestión \& Desarrollo, 9(1), 161-173 (2012)

Palés, J.L., Planificar un currículum o un programa formativo, Educación Médica, 9(2), 59-65 (2006)

Polaino, Cecilia J. y Romillo, Antonio de J., Vinculación con la Sociedad en la Universidad de Otavalo, Ecuador, doi: 10.4067/S0718-50062017000300004, Form. Univ. [online], 10(3), 21-30 (2017)

Quispe, A., Victorino L. y Atriano, R.A., Vinculación de Instituciones de Educación Agrícola Superior (IEAS) con sectores productivos. El caso de la residencia profesional en el Instituto Tecnológico del Altiplano de Tlaxcala (ITAT), Revista de la Educación Superior, 43(170), 135-152 (2014)

Raposo, M. y Zabalza, M.A., La formación práctica de estudiantes universitarios: Repasando el Practicum, Revista de Educación, 354, 17-20 (2011)

Villordo Saucedo, J.A., Las residencias profesionales en la carrera de ingeniería química, https://goo.gl/DYzKVN, ISSN: 1405-5597, Conciencia Tecnológica [en línea], (17), (2001) 
